\title{
Belle II status and Physics prospects
}

\author{
Chiara La Licata*广 \\ Kavli IPMU (WPI) \\ E-mail: chiara.lalicata@ipmu.jp
}

The Belle II experiment at the SuperKEKB energy-asymmetric $e^{+} e^{-}$collider is a substantial upgrade of the $\mathrm{B}$ factory facility at the Japanese KEK laboratory. The design luminosity of the machine is $8 \times 10^{35} \mathrm{~cm}^{-2} \mathrm{~s}^{-1}$ and the Belle II experiment aims to record $50 \mathrm{ab}^{-1}$ of data, a factor of 50 more than its predecessor. With this data set, Belle II will be able to measure the CabibboKobayashi-Maskawa (CKM) matrix, the matrix elements and their phases, with unprecedented precision and explore flavor physics with $B$ and charmed mesons, and $\tau$ leptons. Belle II has also a unique capability to search for low mass dark matter and low mass mediators. We also expect exciting results in quarkonium physics with Belle II. From February to July of this year, the machine has completed a commissioning run, achieved a peak luminosity of $5.5 \times 10^{33} \mathrm{~cm}^{-2} \mathrm{~s}^{-1}$, and Belle II has recorded a data sample of about $0.5 \mathrm{fb}^{-1}$. Regular operations are expected to start in March 2019. In this presentation, we will review the status of the Belle II detector, the results of the commissioning run and the near-term prospects for physics at Belle II.

ALPS 2019 An Alpine LHC Physics Summit

April 22 - 27, 2019

Obergurgl, Austria

\footnotetext{
* Speaker.

${ }^{\dagger}$ for the Belle II Collaboration
} 


\section{Introduction}

The first generation of $\mathrm{B}$ factories, PEP-II and KEKB, and the related experiments BaBar and Belle, operated for 10 years achieving substantial results. Both the two experiments gave significant contributions to B physics finding the first evidence of the CP violation outside the kaon system and the experimental confirmation of the Cabibbo-Kobayashi-Maskawa (CKM) mechanism. Measurements of the parameters of the Unitarity Triangle, and related parameters of the CKM matrix were completed and several constraints were placed on new physic (NP) models. The key of this success lies on a large collected data sample, a clean event structure, the asymmetric beam energy for time-dependent measurements and excellent detector performance. Despite of all these successfully results the existing constraints cannot exclude the existence of non Standard Model physics that can arise in various flavour physics observables. A new generation of flavour factory, SuperKEKB [1] with Belle II [2] detector, aims to extend the search for NP in the flavour sector at the precision frontier using a complementary approach with respect to LHC experiments.

\section{SuperKEKB and Belle II}

SuperKEKB is an asymmetric collider aiming at delivering the highest instantaneous luminosity ever reached $\left(8 \times 10^{35} \mathrm{~cm}^{-1} \mathrm{~s}^{-1}\right.$, about 40 times larger than that provided by KEKB) and to collect $50 \mathrm{ab}^{-1}$ in 8 years of operation. The facility has been designed to collide electrons and positrons at the centre-of-mass energies in the region of the $\Upsilon$ resonances. Most of the data will be collected at the $\Upsilon(4 S)$ resonance that is just above the threshold for B meson pair production. At the designed luminosity it will produce around $5 \times 10^{10} b, c$ and $\tau$ pairs opening possibilities for a wide physical program. There are two essential elements that allow the increase of luminosity: the reduced beam spot size by a factor 20 and an increase in the beam currents by a factor 2 . However with the increase in luminosity we need to face also with the increase of the beam-related background.

The Belle II detector (fig. 1) will operate in a harsher environment with respect to Belle. To cope with the high background maintaining or improving the Belle performance, all the Belle II components have been substantially upgraded or they are new detectors. There are some substantial changes with respect to the Belle detector that will have a relevant impact on the Belle II achievements. The new tracking system, consisting of a Pixel Vertex Detector (PXD), a Silicon strip Vertex Detector (SVD) and a Central Drift Chamber (CDC) will contribute in vertex reconstruction improvements. A better charge track reconstruction and $d E / d x$ measurement will be allowed by a larger central drift chamber built with smaller cells to operate with higher event rates. A time-of-propagation counter and the aerogel ring-imaging Cherenkov detector are used for particle identification with a fake rate lower than in Belle. Finally in the $K_{L}$ and muon detector some RPCs layers have been substituted with scintillators with shorter dead time. The design choices of the Belle II detector translate into improvements on the impact parameter resolution, increase in $K_{s}$ efficiency, a better $K / \pi$ separation and $\pi^{0}$ reconstruction.

\section{Schedule overview}

The first commissioning phase (phase 1) for the SuperKEKB facility has been concluded in 


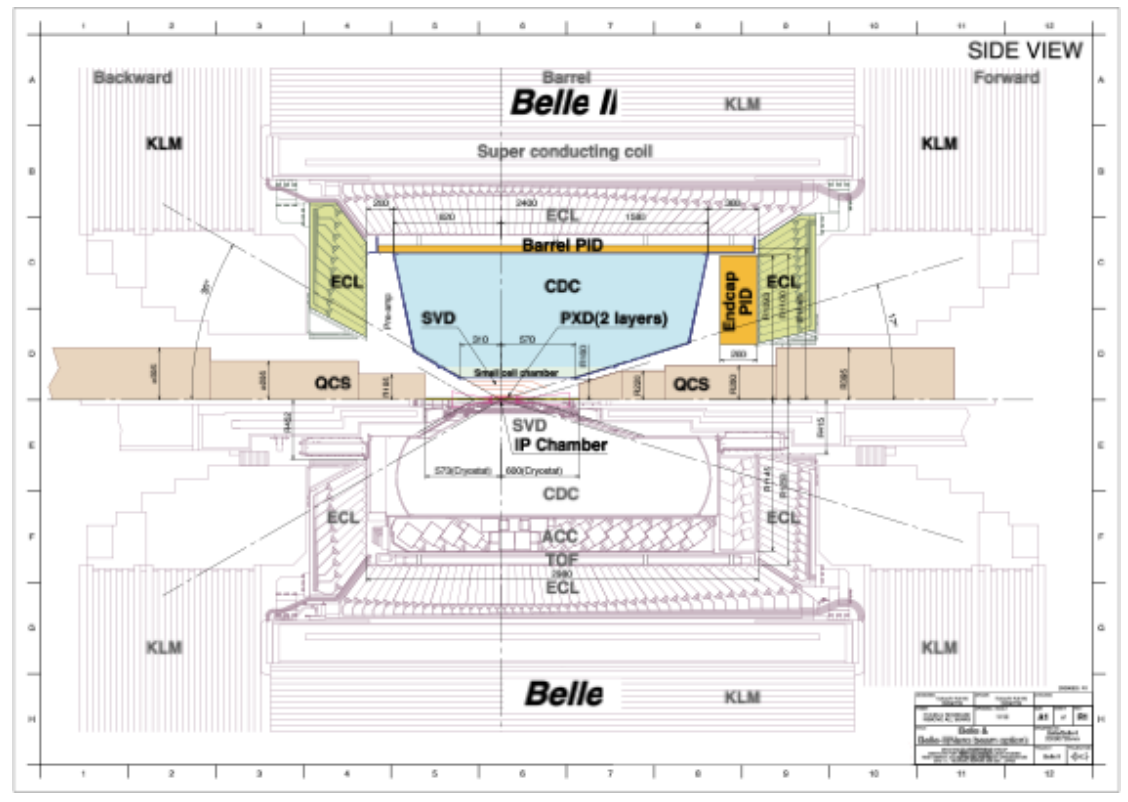

Figure 1: Belle vs Belle II detectors. In the top half of the drawing a side view of the Belle II detector is shown, with new or upgraded components are shown in colours. In the bottom half is the old Belle detector.

June 2016. In this phase no collisions occurred since the machine operated without the final focusing system and without the Belle II detector. Beam storage and tuning, vacuum scrubbing and some optics studies were successfully carried out in that stage. Moreover the first beam-background measurements were performed providing feedback to the accelerator team about the effects of some accelerator parameters on background conditions at the interaction point.

The second phase of commissioning has been concluded last year (July 2018). SuperKEKB with the final focusing system and the Belle II detector, except for the completed vertex detector, operated for 5 months. The focusing system in place allowed collisions achieving the luminosity peak of $5.5 \times 10^{33} \mathrm{~cm}^{-2} \mathrm{~s}^{-1}$.

The Phase 3 physics run started in March 2019 and concluded at the end of June 2019. The full Belle II detector collected around $6.5 \mathrm{fb}^{-1}$ and extensive detector and software performance studies are under completion. The target luminosity of $8 \times 10^{35} \mathrm{~cm}^{-2} \mathrm{~s}^{-1}$ is foreseen to be reached in 2020 .

\section{Phase II achievements}

During phase 2 SuperKEKB operated in collision mode for the first time recording collisions data corresponding to an integrated luminosity of $472 \mathrm{pb}^{-1}$. One of the main purpose of this run was to find the best accelerator conditions performing beam tunings and optics studies. The data collected during phase 2 allowed to reach a better understanding of the beam-background levels and reliably estimate of future background conditions but also to check detector and software performances. The tracking system had a smooth operation since the beginning of the run with tracks available from both VXD and CDC since the start of collisions. At beginning of phase 2 
VXD alignment was determined and validated. Fig. 2 shows the invariant masses of $J / \psi$ (top left) and $K_{S}$ (top right) as examples of charged track reconstruction capabilities in Belle II.

Another example of performance during this run is provided by the fig. 2 (right bottom) that is the $\gamma \gamma$ invariant mass of clusters reconstructed in the electromagnetic calorimeter, showing the $\pi^{0}$ mass peak. Finally fig. 2 (left bottom) is an example of kaon identification capabilities with early calibration and alignment.
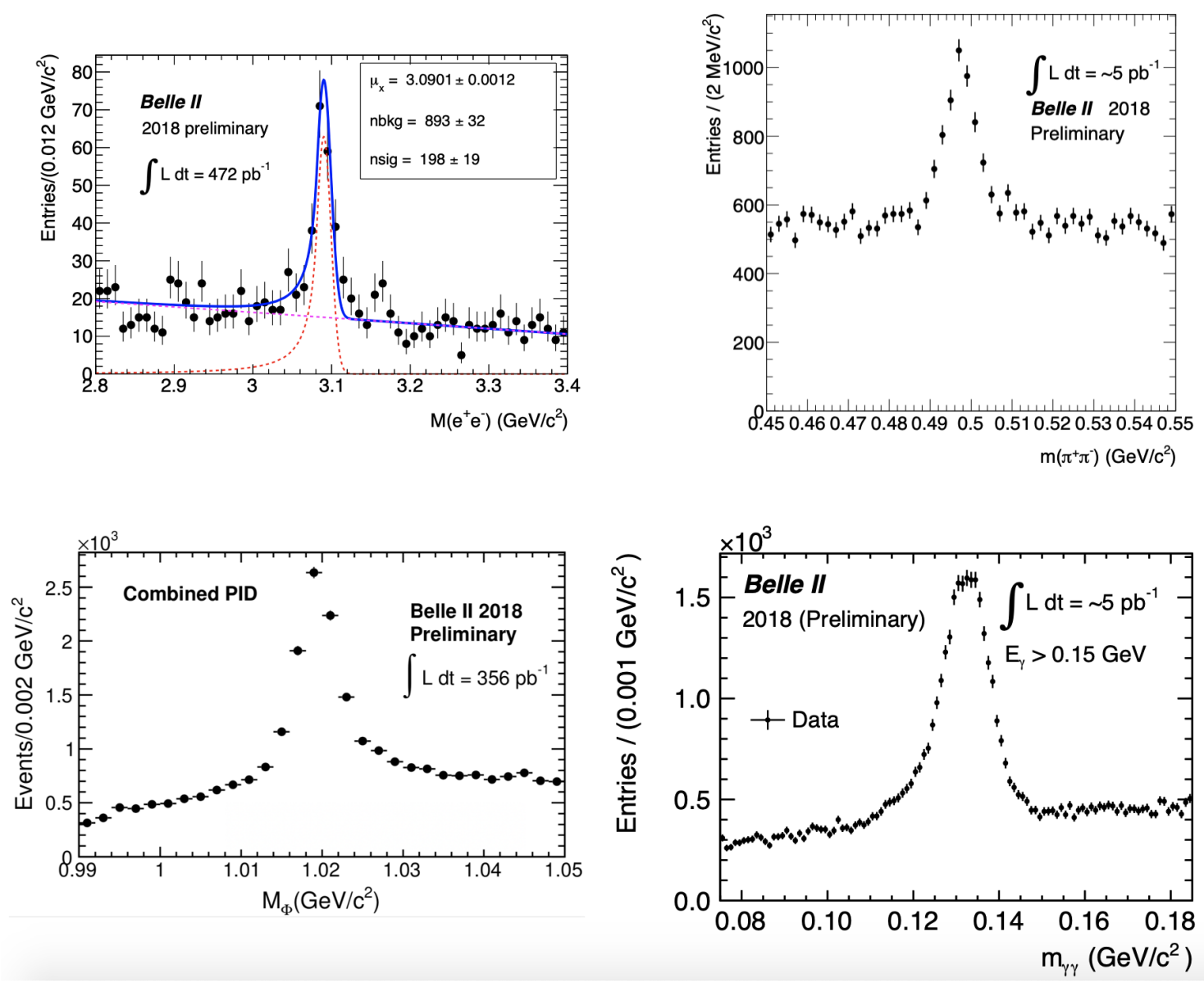

Figure 2: Left top: invariant mass distribution of $J / \psi$ candidates in the $e^{+} e^{-}$final state with $472 \mathrm{pb}^{-1}$ of collision data. Right top: invariant mass distribution of $K_{s} \rightarrow \pi^{+} \pi^{-}$in $5 \mathrm{pb}^{-1}$ of collision data. Left bottom: invariant mass distribution of $\pi^{0} \rightarrow \gamma \gamma$ in $5 \mathrm{pb}^{-1}$ of collision data. Right bottom: invariant mass distribution of $\phi \rightarrow K^{+} K^{-}$with both kaons identified.

The dataset collected during this phase has also been used to perform the first physical studies with the "re-discovery" of known physics process. Vary charm decay modes have been observed validating the potential for charm physics at Belle II. Fig. 3 shows, just as an example, the $D^{0}$ invariant mass reconstructed in the $K^{-} \pi^{+}$final state. A total of $245 B$ signal candidates have been reconstructed considering the $B \rightarrow J \psi K^{*}$ and $\mathrm{B} \rightarrow D^{*} h$ (with $\mathrm{h}=\pi, \rho$ ) modes using $471 p b^{-1}$. Fig. 4 shows the beam mass constrained (left) and the $\Delta E$ (right) distributions. 


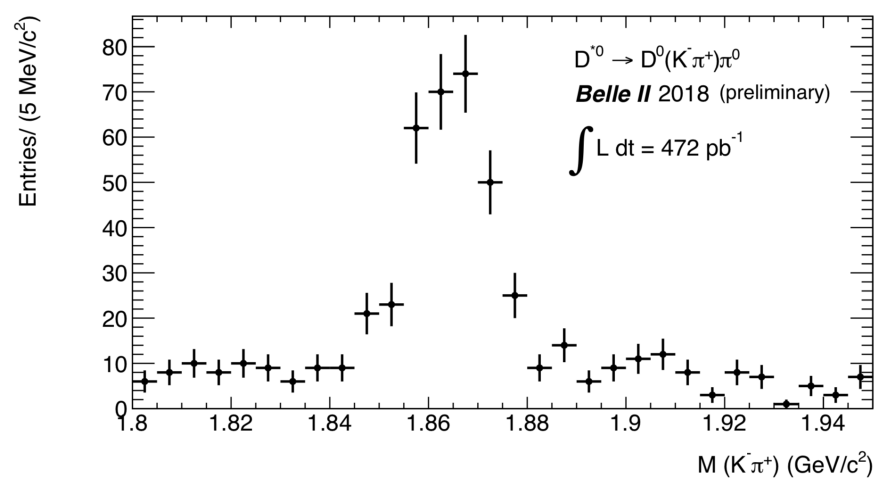

Figure 3: This figure shows the invariant mass distributions of charm candidates in $472 \mathrm{pb}^{-1}$ of collision data, in the mode $D^{* 0} \rightarrow D^{0} p i^{0} D^{0} \rightarrow K^{-} p i^{+}$
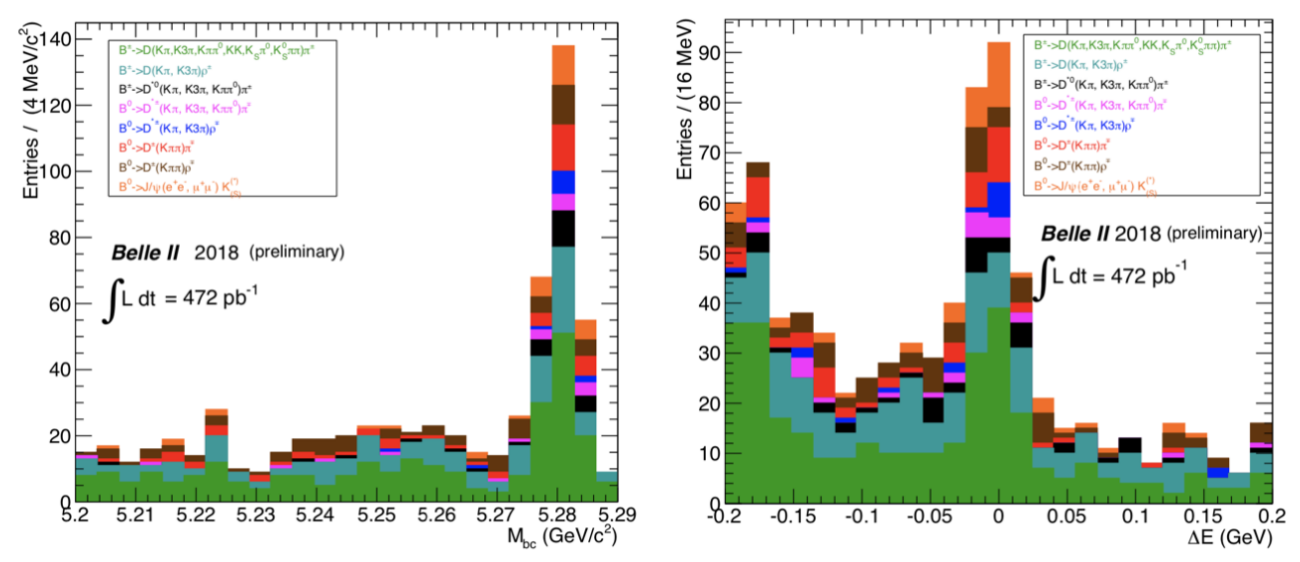

Figure 4: This figure shows the $M_{b c}$ distribution (left) and $\Delta \mathrm{E}$ distribution (right) of $\mathrm{B}$ candidates in 472 $\mathrm{pb}^{-1}$ of collision data, in the modes $\mathrm{B} \rightarrow J / \psi K^{*}$ and $B \rightarrow D^{*} h$ (with $\mathrm{h}=\pi, \rho$ ).

\section{Prospects}

The physics program of Belle II is wide, not only B physics but also charm, $\tau$ and quarkonium physics, electroweak precision measurements and dark sector searches. This paper will focus only on a very restricted sample of topics giving some experimental prospects at Belle II.

\subsection{Time-dependent CP-violating B decays}

The $\mathrm{CP}$ violation in the $\mathrm{B}$ meson system has been clearly established and, although there is a good agreement with expectations of the Standard Model, further measurements are needed to over-constraint the Unitarity Triangle (UT) and search for new physics effects. One of the main strengths of Belle II is that it allows precision measurements of $\mathrm{CP}$ violation (CPV) looking for direct CPV and indirect CPV from interference between the $B \rightarrow f$ and $B \rightarrow \bar{B} \rightarrow f$ mixing amplitude. Precision measurements of the Cabibbo-Kobayashi-Maskawa (CKM) phases $\phi_{1}$ and $\phi_{2}$ are crucial inputs to the CKM unitarity triangle fits. The common strategy to measure the $\phi_{1}$ and $\phi_{2}$ 
angles is the time-dependent $\mathrm{CP}$ violation. The $\mathrm{B}$ factories are the ideal environment for these kind of measurements. The B mesons are produced through the $\Upsilon(4 s)$ decay in an entangled state that evolves coherently. The identification of one of the two produced B mesons allows to determine unambiguously the flavour of the other B. The two key aspects that have to be considered for this technique are the tagging efficiency and the $\Delta t$ resolution where $\Delta t$ is the interval between the time $t_{0}$ at which the flavor of the $B$ meson is observed and the time in which the meson decays into the $\mathrm{CP}$ eigenstate. Dedicated studies have been performed to evaluate the tagging efficiency and the $\Delta t$ resolution. In both these two parameters there is an improvement with respect Belle ones. With the full Belle II data sample, it is possible to improve the unitarity triangle angles measurement to a precision of 1 degree level or less.

\section{$5.2 B \rightarrow D^{(*)} \tau v_{\tau}$ decays}

Relying on a good detector hermeticity and the precisely known initial beam energies, Belle II has unique capability in the reconstruction of final state with neutral particles. The semileptonic $B$ decay involving a $\tau$ lepton can be used as a probe of NP contribution testing the lepton flavour universality. The $B \rightarrow D^{(*)} \tau \nu_{\tau}$ mode is very sensitive to models that predict the existence of charged Higgs $\left(H^{ \pm}\right) . H^{ \pm}$should behave like the charged weak bosons $W$ apart from its couplings to fermions, which are proportional to their masses. The contribution of $H^{ \pm}$is, thus, expected to be largest in $D^{(*)} \tau \nu_{\tau}$ final state due to the masses of the $\tau$ lepton and the $b$ quark.

The ratio defined as:

$$
R\left(D^{(*)}\right)=\frac{B \rightarrow D^{(*)} \tau \nu_{\tau}}{B \rightarrow D^{(*)} l \nu_{\tau}}
$$

with $l=e, \mu$ is a theoretically and experimentally clean observable to test the lepton flavour universality violation, because some theoretical uncertainties and measurement systematics partially cancel out. Belle [3] measured this observable for the first time but then it has been precisely remeasured by Babar [4], Belle [5] and LHCb [6]. The combination of these measurements shows a $3.9 \sigma$ deviation from the SM prediction. The expected precision of these measurements with the full Belle II dataset is shown in fig. 5, compared with current measurements from other experiments and compared to SM expectation. This estimate is based on the existing results from Belle and the expected statistical and experimental improvements at Belle II.

\section{$5.3 B \rightarrow K^{*} l l$}

The decays B to $K^{*}$ are mediated by flavour changing neutral current process $b \rightarrow s l l$. This transition is forbidden at the tree level in the SM and 1 loop diagram is involved. Heavy new particles can contribute to this process, sensitive probe for NP particle search. Also in this case the ratio between $\mathrm{BR}$ of the process in which muons are involved over BR of decay with electrons in the final state is more powerful as some systematic uncertainties can be cancelled out. BaBar and Belle performed this measurement showing consistent results with the SM within large statistical uncertainty while recently the LHCb collaboration [7] showed a tension in this observable. A combined $2.52 \sigma$ deviation from SM expectations has been observed. Further investigation of this discrepancy is thus needed. At Belle II the same detection efficiency is expected for both muons and electrons, thanks to an improved resolution in the ECL measurement of the electron momentum. From simulation studies, Belle II is expected to reach comparable precision to the current $\mathrm{LHCb}$ 


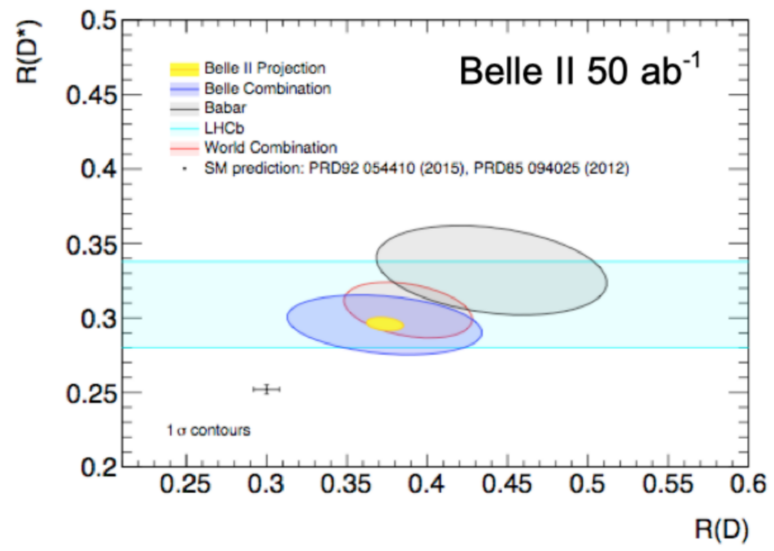

Figure 5: Expected Belle II sensitivity to $\mathrm{R}\left(\mathrm{D}\left(^{*}\right)\right)$ with the full $50 \mathrm{ab}^{-1}$ data sample.

measurement with an integrated luminosity of about $5 \mathrm{ab}^{-1}$ and a accuracy better than $10 \%$ with $10 \mathrm{ab}^{-1}$.

\section{Summary}

The first physics run of Belle II has just successfully concluded and $6.5 \mathrm{fb}^{-1}$ have been collected. The plan is to reach five-fold the Belle sample size in a couple of years and a further factor of ten within the subsequent five years. Important results in relevant measurements in the physics flavour sector are expected thanks to the large dataset together with detector and software performance improvements.

\section{References}

[1] "The Physics of the B Factories", THE EUROPEAN PHYSICAL JOURNAL. C, PARTICLES AND FIELDS, November 2014, 74:3026, doi: 10.1140/epjc/s10052- 014-3026-9.

[2] Belle II Technical Design Report, KEK Report 2010-1. Available: http://www-superkekb.kek.jp/documents/B2TDR.pdf

[3] A. Matyja et al. (Belle Collaboration), "Observation of $B^{0} \rightarrow D^{*-} \tau^{+} v_{\tau}$ Decay at Belle", Phys. Rev. Lett., 99, 191807 (2007), arXiv:0706.4429

[4] J. P. Lees et al. (BaBar Collaboration), "Evidence for an Excess of $\bar{B} \rightarrow D^{(*)} \tau^{-} \bar{v}_{\tau}$ Decays", Phys. Rev. Lett., 109, 101802 (2012), arXiv:1205.5442

[5] M. Huschle et al. (Belle Collaboration), "Measurement of the branching ratio of $\bar{B} \rightarrow D^{(*)} \tau^{-} \bar{v}_{\tau}$ relative to $\bar{B} \rightarrow D^{(*)} \ell^{-} \bar{v}_{\ell}$ decays with hadronic tagging at Belle", Phys. Rev., D92(7), 072014 (2015), arXiv: 1507.03233

[6] R. Aaij et al. (LHCb Collaboration),"Measurement of the Ratio of Branching Fractions $\mathscr{B}\left(\bar{B}^{0} \rightarrow D^{*+} \tau^{-} \bar{v}_{\tau}\right) / \mathscr{B}\left(\bar{B}^{0} \rightarrow D^{*+} \mu^{-} \bar{v}_{\mu}\right)$ ", Phys. Rev. Lett., 115(11), 111803, [Addendum: Phys. Rev. Lett. 115, no.15, 159901 (2015)] (2015), arXiv:1506.08614.

[7] Aaij, Roel and others (LHCb Collaboration), "Search for lepton-universality violation in $B^{+} \rightarrow K^{+} \ell^{+} \ell^{-}$decays", Phys. Rev. Lett., 122, no. 19, 191801 (2019), arXiv:1903.09252. 